\title{
Using a joint spectral fitting technique to explore the outflow geometry of GX 339-4 during the hard state
}

\author{
Riley Connors* \\ Anton Pannekoek Institute for Astronomy, University of Amsterdam, The Netherlands \\ E-mail: r.m.t.connors Quva.nl
}

\section{David van Eijnatten}

Anton Pannekoek Institute for Astronomy, University of Amsterdam, The Netherlands

E-mail: d.vaneijnatten@student.uva.nl

\section{Chiara Ceccobello}

Anton Pannekoek Institute for Astronomy, University of Amsterdam, The Netherlands

E-mail: c.ceccobello@uva.nl

\section{Sera Markoff}

Anton Pannekoek Institute for Astronomy, University of Amsterdam, The Netherlands

E-mail: s.b.markoffeuva.nl

Black hole low-mass X-ray binaries (LMXBs) are transient in nature, such that their luminosities and spectra evolve significantly over periods of weeks to months. Such changes are due to physical alterations to the structure of the accretion inflow and gaseous outflows. LMXBs spend most of their time in a hard state (wherein their X-ray spectra are power laws and thus dominated by higher energies), with order-of-magnitude variations in luminosity. A key issue in physically characterising this state is finding a way to dissect the components contributing to this power law emission: this has up to now proved challenging due to degeneracies in spectral modelling. Solving this issue of modelling degeneracy is vital in the broader context of understanding the physics of accretion around black holes, solar mass and supermassive alike- this is a key factor in the fields of active galactic nuclei feedback and supermassive black hole growth. It is also required for the determination of black hole spin via X-ray reflection modelling, since the results of such studies depend on the assumed geometry of the irradiator. In order to disentangle these components, one needs to invoke all the available spectral and timing information, and build a self-consistent physical picture. I will present work we have been doing on attempting to break modelling degeneracies by fitting a semi-analytical outflow-dominated model to broadband observations of GX 339-4 from radio to X-ray, tracking the evolution of key model parameters with the X-ray variability properties of the source. We jointly model the broadband spectra during states of similarity in variability properties, and find key best fit parameters hold indistinguishable values. I will discuss further work we are doing to advance X-ray reflection modelling by adding X-ray emission from the jet to current models.

11th INTEGRAL Conference Gamma-Ray Astrophysics in Multi-Wavelength Perspective 10-14 October 2016

Amsterdam, The Netherlands

* Speaker. 


\section{Introduction}

Black hole X-ray binaries with low-mass companions (LMXBs) exhibit a wealth of well, but not wholly understood, spectral and timing phenomena as they evolve through regular outburst cycles $[1,2,3,4]$. Despite the evidently complicated evolution of the spectral and timing properties of LMXBs, we are able to use some basic classifications to distinguish different states of the systems. The two simplest yet non-encompassing catergories are soft and hard states, given based on the hardness of the X-ray spectrum (the ratio of X-ray counts in a harder energy band, typically $>$ a few to tens of $\mathrm{keV}$, to X-ray counts in a softer energy band, typically 0.1 to $<$ a few $\mathrm{keV})$. LMXBs in the soft state are dominated by a multi-temperature blackbody spectrum from an optically thick, geometrically thin accretion disc [5]. LMXBs in the hard state are dominated by a hard power law spectrum, but the origin of the emission is less certain. Candidates for emission mechanisms which can produce this power-law spectrum include inverse Compton scattering of disc photons by hot electrons in an optically thin 'corona', synchrotron self-Compton scattering within a jet/outflow (SSC), or optically thin synchrotron emission within a jet/outflow [6, 7, 8, 9]. Multiwavelength observing campaigns allow us to make progress in disentangling these components, as well as time-variability studies, where the rms variability and time lag estimates allow us to predict the size and location of the emission regions [2].

Radio and X-ray luminosity correlations indicate an intimate physical connection between the $\mathrm{X}$-ray emitting region close to the black hole, and the jet radio emission $\geq 10^{4} r_{g}$ away from the black hole $[10,11,12,13,14,15,16,17]$. When we consider also the information on structure evolution in the X-ray emitting regions which comes from time-variability studies, we can further understand this connection. Fourier-domain studies of the X-ray variability of LMXBs show low-rms variability in soft states, and high rms-variability in hard states. Sharp features seen in the power-spectrum, known as quasi-periodic oscillations (QPOs), point to strongly variable components in narrow frequency bands, currently best explained by precessing structures in the inner regions of the accretion flow $[18,19]$. The evolution of the broadband rms noise is best explained by mass accretion rate fluctuations propagating through the flow on viscous timescales, giving rise to the characteristic shape of the power spectrum [20].

[21] developed a novel state classification method of LMXB states which characterises their frequency-dependent variability throughout the course of an outburst. A single variable, known as the power-colour hue, represents the shape of the power spectrum, given by an angular position on an annulus in a plot of one power-spectral ratio against another-shown in Figure 1. One can use this information to track spectral parameters alongside timing characteristics.

In this work we investigate how the physical and spatial characteristics of the jet in GX 339-4 evolve alongside its changing time-variability properties. GX339-4 is a LMXB of great interest due to the multiple outburst observations we have. It has an orbital period of $\sim 1.7$ days [22], lies a distance of 6-15 kpc away [23] (with an optimal distance of $\sim 8 \mathrm{kpc}$ [24]), and the most recent measurement of its mass function predicts $\sim 1.91 \pm 0.08 M_{\odot}$ (Heida et al., in preparation).

GX 339-4 has a compact radio jet during the hard state [25], and the emission from this jet dominates down to IR and likely optical wavelengths [26, 27, 28]. Correlations between the optical/NIR/X-ray light curves during various GX 339-4 outbursts indicate a physical connection between the inner accretion flow/corona/jet and the outer regions of the jet at $\sim 10^{3}-10^{4} r_{g}$. GX 
339-4 has simultaneous NIR, optical and X-ray quasi-periodic oscillation (QPO) detections at 0.08 , 0.08 , and $0.16 \mathrm{~Hz}$ frequencies respectively [29]. Its IR and X-ray variability are strongly correlated, with NIR photons lagging X-ray photons by $>100 \mathrm{~ms}$. This points to a physical picture in which variations propagating through the accretion flow towards the black hole subsequently propagate through the jet, giving rise to delayed NIR variability.

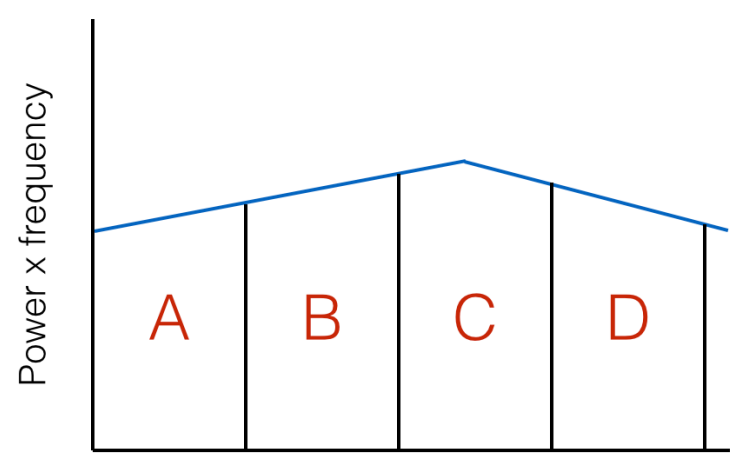

Frequency

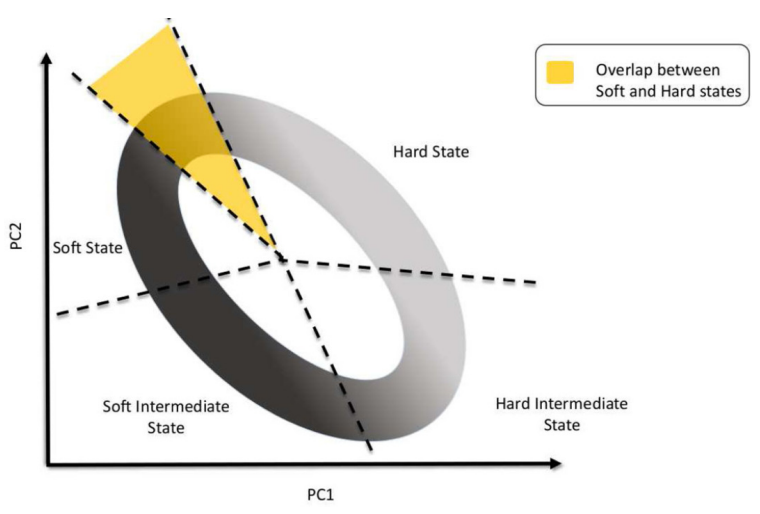

PC1

Figure 1: Left: A conceptual plot showing how [21] integrate over four separate frequency bands in the power spectra of their LMXB sample, taking the ratios PC1 = band C / band A, PC2 = band D / band B. Right: The plot (taken from [21]) which follows from this analysis, allowing one to identify the variability state of a LMXB by its angular position on the annulus, synonymous with hardness-intensity diagrams.

\section{Broadband modelling}

We model 20 separate quasi-simultaneous broadband observations of GX 339-4, covering a 15-year period (1997-2012). Radio data are taken from observations made with Australian Telescope Compact Array (ATCA) [15], optical and near-infrared (NIR) data are taken from observations made with the SMARTS 1.3 m telescope $(V, J, I$, and $H$ bands-dereddened using the extinction law of [30] with $E(B-V)=0.94 \pm 0.19$ [31], such that $A_{V}=2.9 \pm 0.6$.) [32], and archival X-ray data taken from observations made with the Rossi X-ray Timing Explorer (RXTE). Observations are all contemperaneous to within a 24-hour window.

We use a semi-analytical, zonal jet model (see [9, 33, 34, 35]) based on early work by [36]. The model reproduces a broadband spectrum from radio-to-X-ray frequencies via several emission mechanisms: thermal and non-thermal synchrotron (due to thermal and accelerated electrons respectively) in the optically thin and thick regimes, synchrotron self-Compton emission due to inverse Compton scattering by synchrotron emitting electrons and of disc photons, and a multitemperature disc blackbody spectrum. The inverse Compton scattering treatment is a multiple scattering routine, described in detail in Ceccobello et al. (in preparation). Table 1 shows the key parameters and their physical descriptions.

\section{Fitting method}

All model fits are performed using the multiwavelength data analysis package IS IS [37], and all models are forward-folded through the detector response matrices. The responses of all X-ray 
Table 1: A list of the main input parameters of the agnjet model

\begin{tabular}{cl}
\hline Parameter & Description \\
\hline$N_{j}$ & the normalised jet power, in units of $L_{\mathrm{Edd}}$ - \\
$r_{0}\left(r_{g}\right)$ & the radius of the jet nozzle. \\
$\Theta_{e}\left(k T_{e} / m c^{2}\right)$ & the electron temperature of the input distribution. \\
$k$ & the ratio of magnetic to electron energy density, $U_{B} / U_{e}$, otherwise known as \\
& the partition factor. \\
the power-law index of the accelerated electron distribution. & the distance from the black hole along the jet axis where particle acceleration \\
$z_{a c c}\left(r_{g}\right)$ & begins. \\
$n_{n t h}$ & the fraction of particles accelerated at a distance $z_{a c c}$ from the black hole along \\
& the axis of the jet. \\
$f_{s c}$ & $\beta_{s h}^{2} /\left(\lambda / R_{\text {gyro }}\right)$ where $\beta_{s h}$ is the shock speed relative to the plasma, $\lambda$ is the \\
& scattering mean free path in the plasma at the shock region, and $R_{g y r o}$ is the \\
& gyroradius of the particles in the magnetic field. In reality we do not require \\
& a shock so this parameterisation can generally be seen as a measure of the \\
& acceleration efficiency.
\end{tabular}

spectra correspond to those of the two X-ray instruments, the Proportional Counter Array (PCA) and the High Energy X-ray Transmission Spectrometer (HEXTE), whereas for the radio and NIRoptical data we assign "dummy" responses equivalent to a detector of effective area $=1 \mathrm{~m}^{2}$-they are loaded as flux measurements.

We model all broadband spectra energy distributions (SEDs) of GX 339-4 as the sum of absorbed, reflected jet and coronal components and a Gaussian Iron $\mathrm{K} \alpha$ line: tbabs $\times$ reflect(agnjet + nthcomp + gaussian), where agnjet represents the jet component, and nthcomp represents a spherical corona in the inner regions of the accretion flow [38, 39]. The agnjet model includes a coronallike jet base which produces an SSC spectrum, but the key distinctions between this component and a classical corona are 1) that the primary input photon distribution for scattering is synchrotron emission from the jet, peaking in the optical bands, and 2) the electrons are treated purely relativistically, and thus have $\Theta_{e} \equiv k T_{e} / m c^{2}>511 \mathrm{keV}$, whereas typical coronae temperatures are $\sim 50-200$ $\mathrm{keV}$. This results in more curvature in the SSC spectrum, such that pure jet SSC emission is unlikely to reproduce power-law spectra from a few $\mathrm{keV}$ up to $100 \mathrm{~s}$ of $\mathrm{keV}$.

\section{Preliminary fitting results and conclusions}

Here we show some preliminary model fitting results, with a full description to follow in a forthcoming paper (Connors et al., in preparation). Figure 2 shows two individual fits to two separate GX 339-4 broadband datasets. Key best fit parameters are also shown (though without confidence limits), and we can say at least provisionally that there is no fundamental change to the jet structure-a full markov chain monte carlo parameter exploration must be carried out in order to ascertain the significance of the changes in parameters. In both cases the dominant emission component in the X-ray is SSC, where the SSC can be seen to cut off, failing to reproduce a 
power law up to the highest X-ray energies seen in the data. The discrepancy is accounted for by a high reflection fraction, such that the Compton hump (due to Compton scattering of the high energy X-rays off cold electrons in the disc) contributes to approximate a power law spectrum, with some contribution at higher X-ray energies from up-scattered disc photons. It is unlikely that such a scenario produces an un-interrupted power law spectrum through the hard state of GX 3394. The early cut-off is due to the naturally high electron temperatures in our modelling, since the electrons are solely relativistic - higher-order SSC components peak at energies at least an order of magnitude beyond that of the 0th-order peak, producing a curvy spectrum with multiple peaks. Coronal IC models on the other hand treat the electrons non-relativistically, with typical electron temperature $\leq 150 \mathrm{keV}$. We will improve our treatment of the jet dynamics and radiation spectrum in future works to include a trans-relativistic electron population, and investigate the effects of electron heating on the resultant spectrum and the variability we may expect to observe.

\section{X-ray reflection of jet emission}

As shown by [40], the geometry of the X-ray irradiator in studies of reflection of LMXB accretion discs (and indeed accretion discs in all accreting systems for which the disc sits within $\sim 100 r_{g}$ of the compact object) is an important component to consider when performing such spectral modelling (e.g. [41, 42, 43, 44, 45, 46]). However, what's clear is that the geometry of the irradiator must also be understood in terms of the spectral shape variations over space and timei.e. whether the irradiator is a coronal region close to the black hole, or an extended emission region resembling a jet, the key to relating this to reflection is to understand the physics of these regions. We are working on incorporating a jet (SSC) component into current reflection models, where we can properly track how the physical state of the jet affects the reflection model's spectral components (the Iron emission line and its broadening, and the Compton hump) (van Eijnatten et al., in preparation). The geometrical factor, which defines how much of the emitted X-ray radiation strikes the disc, is significantly altered when we consider such an extended geometry. We propose that spectral shape variations with height, due to both the extended geometry and electron heating, are vital for interpreting the output of reflection modelling and thus also important if we to achieve better estimates of black hole spin.

\section{References}

[1] M. A. Nowak, Toward a Unified View of Black-Hole High-Energy States, 107 (Dec., 1995) 1207, [astro-ph/9509046].

[2] M. van der Klis, Rapid aperiodic variability in X-ray binaries., X-ray Binaries (1995) 252-307.

[3] R. A. Remillard and J. E. McClintock, Active X-ray States of Black Hole Binaries: Current Overview, in American Astronomical Society Meeting Abstracts, vol. 38 of Bulletin of the American Astronomical Society, p. 903, Dec., 2006.

[4] T. M. Belloni, States and Transitions in Black Hole Binaries, in Lecture Notes in Physics, Berlin Springer Verlag (T. Belloni, ed.), vol. 794 of Lecture Notes in Physics, Berlin Springer Verlag, p. 53, Mar., 2010, 0909.2474 , DOI. 

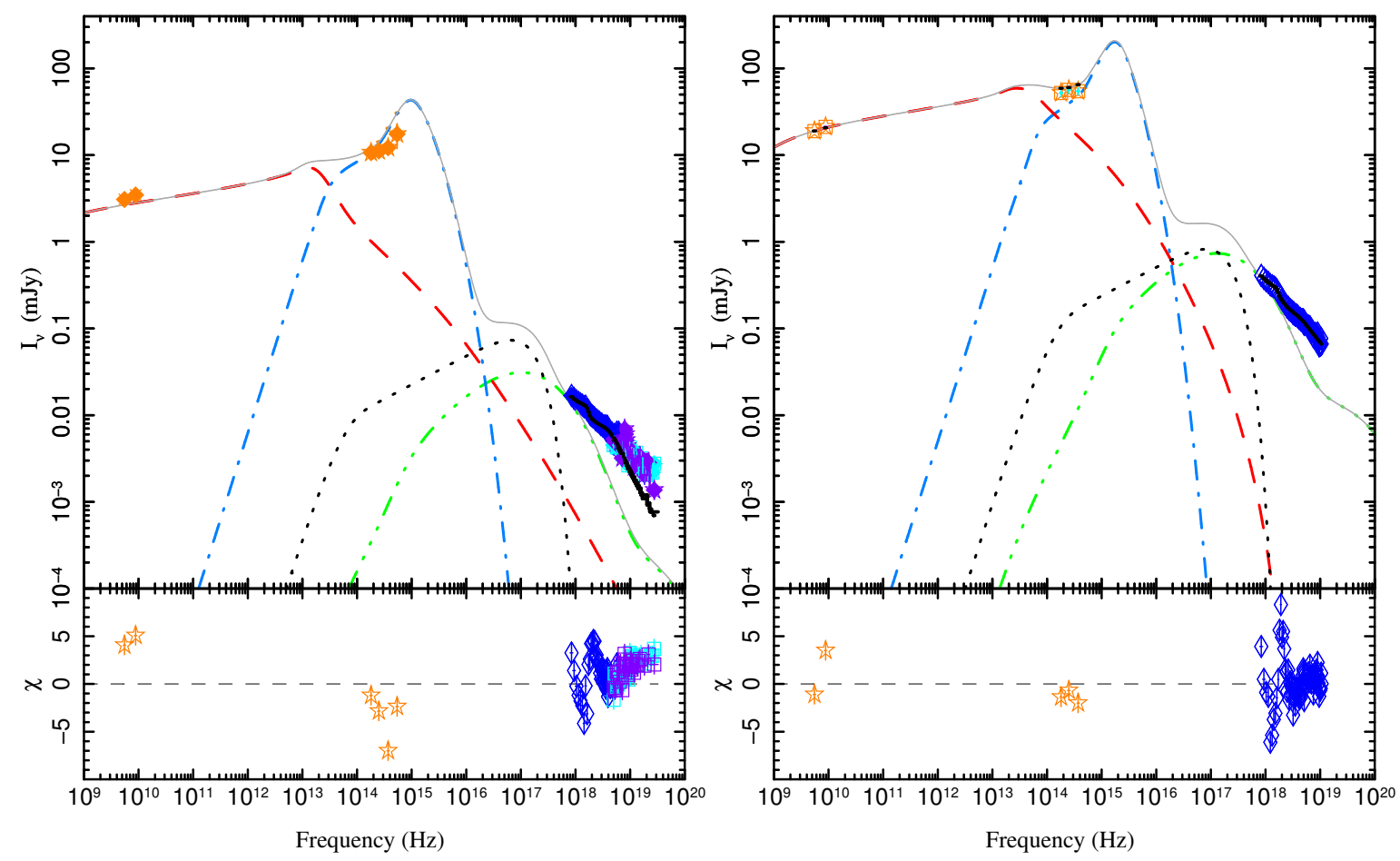

Figure 2: Model fits to broadband spectra of GX 339-4 during its 2005 outburst decay (left: MJD = 53489) and its 2010 outburst rise (right: MJD = 55290). Orange data points are radio and NIR-Optical fluxes from ATCA and SMARTS observations respectively. Dark blue data points are RXTE observations with PCA (binned at minimum $S / N=4.5$ between 3-45 keV), light blue/purple points are RXTE detections with clusters A and B of HEXTE (binned at minimum $S / N=4.5$ between 20-200 keV). All data are shown unfolded from the detector response, whereas residuals are raw data counts - forward-folded model counts. In both panels the model components are as follows: pre-particle-acceleration synchrotron (blue-dot-dashed line), post particle acceleration synchrotron (red dashed line), synchrotron self-Compton (green triple-dotdashed line), and multitemperature disc blackbody (black dotted line). The model fit is shown with black solid lines. In both fits the location in which particles are accelerated into a power law distribution is fixed at $\log z_{a c c}=3.5$. In the left hand panel the best fit solution has $r_{0} \sim 80 r_{g}, N_{j} \sim 0.01, \Theta_{e} \sim 4$, and $k \sim 37$, and in the right hand panel the same parameters are $r_{0} \sim 100 r_{g}, N_{j} \sim 0.04, \Theta_{e} \sim 3$, and $k \sim 20$.

[5] N. I. Shakura and R. A. Sunyaev, Black holes in binary systems. Observational appearance., 24 (1973) 337-355.

[6] A. P. Lightman and D. M. Eardley, Black Holes in Binary Systems: Instability of Disk Accretion, 187 (Jan., 1974) L1.

[7] D. M. Eardley, A. P. Lightman and S. L. Shapiro, Cygnus X-1 - A two-temperature accretion disk model which explains the observed hard X-ray spectrum, 199 (Aug., 1975) L153-L155.

[8] S. L. Shapiro, A. P. Lightman and D. M. Eardley, A two-temperature accretion disk model for Cygnus X-1 - Structure and spectrum, 204 (Feb., 1976) 187-199.

[9] S. Markoff, M. A. Nowak and J. Wilms, Going with the Flow: Can the Base of Jets Subsume the Role of Compact Accretion Disk Coronae?, 635 (Dec., 2005) 1203-1216, [astro-ph/ 0509028 ].

[10] D. C. Hannikainen, R. W. Hunstead, D. Campbell-Wilson and R. K. Sood, MOST radio monitoring of GX 339-4, 337 (Sept., 1998) 460-464, [a stro-ph / 9805332 ]. 
[11] S. Corbel, R. P. Fender, A. K. Tzioumis, M. Nowak, V. McIntyre, P. Durouchoux et al., Coupling of the $X$-ray and radio emission in the black hole candidate and compact jet source GX 339-4, 359 (July, 2000) 251-268, [astro-ph/0003460].

[12] S. Corbel, M. A. Nowak, R. P. Fender, A. K. Tzioumis and S. Markoff, Radio/X-ray correlation in the low/hard state of GX 339-4, 400 (Mar., 2003) 1007-1012, [a stro-ph/0301436].

[13] E. Gallo, R. P. Fender and G. G. Pooley, A universal radio-X-ray correlation in low/hard state black hole binaries, 344 (Sept., 2003) 60-72, [astro-ph/0305231].

[14] S. Corbel, E. Koerding and P. Kaaret, Revisiting the radio/X-ray flux correlation in the black hole V404 Cyg: from outburst to quiescence, 389 (Oct., 2008) 1697-1702, [0806.3079].

[15] S. Corbel, M. Coriat, C. Brocksopp, A. K. Tzioumis, R. P. Fender, J. A. Tomsick et al., The 'universal' radio/X-ray flux correlation: the case study of the black hole GX 339-4, 428 (Jan., 2013) 2500-2515, [1211.1600].

[16] J. C. A. Miller-Jones, P. G. Jonker, T. J. Maccarone, G. Nelemans and D. E. Calvelo, A Deep Radio Survey of Hard State and Quiescent Black Hole X-Ray Binaries, 739 (Sept., 2011) L18, [1106.0097].

[17] E. Gallo, J. C. A. Miller-Jones, D. M. Russell, P. G. Jonker, J. Homan, R. M. Plotkin et al., The radio/X-ray domain of black hole X-ray binaries at the lowest radio luminosities, 445 (Nov., 2014) 290-300, [1408.3130].

[18] C. Done, M. Gierliński and A. Kubota, Modelling the behaviour of accretion flows in X-ray binaries. Everything you always wanted to know about accretion but were afraid to ask, 15 (Dec., 2007) 1-66, [0708.0148].

[19] A. Ingram and C. Done, A physical model for the continuum variability and quasi-periodic oscillation in accreting black holes, 415 (Aug., 2011) 2323-2335, [1101.2336].

[20] Y. E. Lyubarskii, Flicker noise in accretion discs, 292 (Dec., 1997) 679.

[21] L. M. Heil, P. Uttley and M. Klein-Wolt, Power colours: simple X-ray binary variability comparison, mnras 448 (Apr., 2015) 3339-3347, [1405.2024].

[22] R. I. Hynes, D. Steeghs, J. Casares, P. A. Charles and K. O’Brien, Dynamical Evidence for a Black Hole in GX 339-4, 583 (Feb., 2003) L95-L98, [astro-ph/0301127].

[23] R. I. Hynes, D. Steeghs, J. Casares, P. A. Charles and K. O'Brien, The Distance and Interstellar Sight Line to GX 339-4, 609 (July, 2004) 317-324, [a stro-ph/ 0402408 ].

[24] A. A. Zdziarski, M. Gierliński, J. Mikołajewska, G. Wardziński, D. M. Smith, B. A. Harmon et al., GX 339-4: the distance, state transitions, hysteresis and spectral correlations, 351 (July, 2004) 791-807, [astro-ph/ 0402380$]$.

[25] R. P. Fender, Powerful jets from black hole X-ray binaries in low/hard X-ray states, 322 (Mar., 2001) 31-42, [astro-ph/0008447].

[26] P. Gandhi, K. Makishima, M. Durant, A. C. Fabian, V. S. Dhillon, T. R. Marsh et al., Rapid optical and X-ray timing observations of GX 339-4: flux correlations at the onset of a low/hard state, 390 (Oct., 2008) L29-L33, [0 807 . 1529].

[27] P. Gandhi, V. S. Dhillon, M. Durant, A. C. Fabian, A. Kubota, K. Makishima et al., Rapid optical and X-ray timing observations of GX339-4: multicomponent optical variability in the low/hard state, 407 (Oct., 2010) 2166-2192, [1005.4685]. 
[28] P. Casella, T. J. Maccarone, K. O’Brien, R. P. Fender, D. M. Russell, M. van der Klis et al., Fast infrared variability from a relativistic jet in GX 339-4, 404 (May, 2010) L21-L25, [1 002.1233 ].

[29] M. Kalamkar, P. Casella, P. Uttley, K. O’Brien, D. Russell, T. Maccarone et al., Detection of the first infra-red quasi-periodic oscillation in a black hole X-ray binary, 460 (Aug., 2016) 3284-3291, [1510.08907].

[30] J. A. Cardelli, G. C. Clayton and J. S. Mathis, The relationship between infrared, optical, and ultraviolet extinction, 345 (Oct., 1989) 245-256.

[31] P. Predehl and J. H. M. M. Schmitt, X-raying the interstellar medium: ROSAT observations of dust scattering halos., 293 (Jan., 1995) 889-905.

[32] M. M. Buxton, C. D. Bailyn, H. L. Capelo, R. Chatterjee, T. Dinçer, E. Kalemci et al., Optical and Near-infrared Monitoring of the Black Hole X-Ray Binary GX 339-4 during 2002-2010, 143 (June, 2012) 130, [1203.5700].

[33] D. Maitra, S. Markoff, C. Brocksopp, M. Noble, M. Nowak and J. Wilms, Constraining jet/disc geometry and radiative processes in stellar black holes XTE J1118+480 and GX 339-4, 398 (Oct., 2009) 1638-1650, [0904.2128].

[34] R. M. T. Connors, S. Markoff, M. A. Nowak, J. Neilsen, C. Ceccobello, P. Crumley et al., Mass-scaling as a method to constrain outflows and particle acceleration from low-luminosity accreting black holes, ArXiv e-prints (Dec., 2016), [1612.00953].

[35] P. Crumley, C. Ceccobello, R. M. T. Connors and Y. Cavecchi, The jet-disk symbiosis without maximal jets: 1-D hydrodynamical jets revisited, ArXiv e-prints (Mar., 2017), [1703. 02842 ].

[36] H. Falcke and P. L. Biermann, The jet-disk symbiosis. I. Radio to X-ray emission models for quasars., 293 (Jan., 1995) 665-682, [astro-ph/9411096].

[37] J. C. Houck and L. A. Denicola, ISIS: An Interactive Spectral Interpretation System for High Resolution X-Ray Spectroscopy, in Astronomical Data Analysis Software and Systems IX (N. Manset, C. Veillet and D. Crabtree, eds.), vol. 216 of Astronomical Society of the Pacific Conference Series, p. $591,2000$.

[38] A. A. Zdziarski, W. N. Johnson and P. Magdziarz, Broad-band $\gamma$-ray and X-ray spectra of NGC 4151 and their implications for physical processes and geometry., 283 (Nov., 1996) 193-206, [astro-ph/9607015].

[39] P. T. Życki, C. Done and D. A. Smith, The 1989 May outburst of the soft X-ray transient GS 2023+338 (V404 Cyg), 309 (Nov., 1999) 561-575, [astro-ph/9904304].

[40] T. Dauser, J. Garcia, J. Wilms, M. Böck, L. W. Brenneman, M. Falanga et al., Irradiation of an accretion disc by a jet: general properties and implications for spin measurements of black holes, 430 (Apr., 2013) 1694-1708, [1301 . 4922].

[41] T. Dauser, J. Wilms, C. S. Reynolds and L. W. Brenneman, Broad emission lines for a negatively spinning black hole, 409 (Dec., 2010) 1534-1540, [1007.4937].

[42] J. García and T. R. Kallman, X-ray Reflected Spectra from Accretion Disk Models. I. Constant Density Atmospheres, 718 (Aug., 2010) 695-706, [1006.0485].

[43] J. García, T. R. Kallman and R. F. Mushotzky, X-ray Reflected Spectra from Accretion Disk Models. II. Diagnostic Tools for X-ray Observations, 731 (Apr., 2011) 131, [1101.1115]. 
[44] J. García, T. Dauser, C. S. Reynolds, T. R. Kallman, J. E. McClintock, J. Wilms et al., X-Ray Reflected Spectra from Accretion Disk Models. III. A Complete Grid of Ionized Reflection Calculations, 768 (May, 2013) 146, [1303.2112].

[45] T. Dauser, J. García, M. L. Parker, A. C. Fabian and J. Wilms, The role of the reflection fraction in constraining black hole spin, 444 (Oct., 2014) L100-L104, [14 08 . 2347].

[46] J. García, T. Dauser, A. Lohfink, T. R. Kallman, J. F. Steiner, J. E. McClintock et al., Improved Reflection Models of Black Hole Accretion Disks: Treating the Angular Distribution of X-Rays, 782 (Feb., 2014) 76, [1312.3231]. 